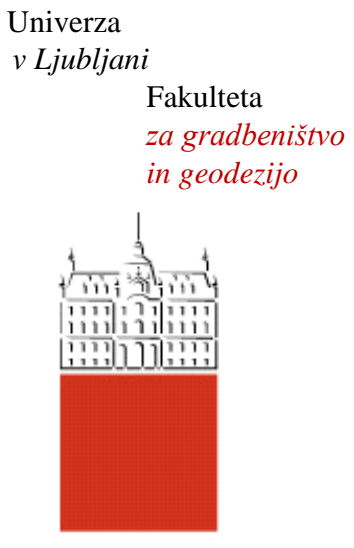

Jamova cesta 2

1000 Ljubljana, Slovenija

http://www3.fgg.uni-lj.si/

\section{DRUGG - Digitalni repozitorij UL FGG http://drugg.fgg.uni-lj.si/}

Ta članek je avtorjeva zadnja recenzirana različica, kot je bila sprejeta po opravljeni recenziji.

Prosimo, da se pri navajanju sklicujte na bibliografske podatke, kot je navedeno:
University
of Ljubljana

Faculty of Civil and Geodetic Engineering

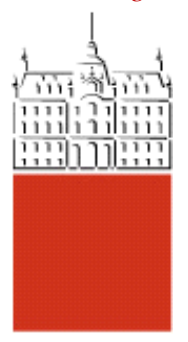

Jamova cesta 2 SI - 1000 Ljubljana, Slovenia http://www3.fgg.uni-lj.si/en/

DRUGG - The Digital Repository http://drugg.fgg.uni-lj.si/

This version of the article is author's manuscript as accepted for publishing after the review process.

When citing, please refer to the publisher's bibliographic information as follows:

Zupan, D., Saje, M. 2005. Analytical integration of stress field and tangent material moduli over concrete cross-sections. Computers \& Structures 83,28-30: 2368-2380. DOI: 10.1016/j.compstruc.2005.03.030. 


\title{
Analytical Integration of Stress Field and Tangent Material Moduli Over Concrete Cross-Sections
}

\author{
D. Zupan and M. Saje* \\ University of Ljubljana, Faculty of Civil and Geodetic Engineering, \\ Jamova 2, SI-1115 Ljubljana, Slovenia
}

\begin{abstract}
This paper presents a novel stress field and tangent material moduli integration procedure over a cross-section of a biaxially loaded concrete beam. The procedure assumes a sufficiently simple analytical form of the constitutive law of concrete, the polygonal shape of the boundary of the simply- or multi-connected cross-section and the monotonically increasing loading. The area integrals are transformed into the boundary integrals and then integrated analytically. The computational efficiency of the procedure is analyzed by comparing it with respect to the number of floatingpoint operations needed in various numerical integration-based methods. It is found that the procedure is not only exact, but also computationally effective.
\end{abstract}

Key words: reinforced concrete section, biaxial bending, analytical integration.

\section{Introduction}

The non-linear finite-element analysis of reinforced concrete spatial beams and frame structures requires the integration of stresses and tangent material moduli over the cross-section. Since the governing equations of these structures are non-linear and must therefore be solved iteratively, the integrals over the cross-sections need to be evaluated many times. Thus, it is of great importance for us to be able to evaluate cross-sectional integrals as efficiently as possible. Since the area of the reinforcing steel bars is relatively small compared to the area of concrete, we may assume a constant stress field across each steel bar, which makes the integration over the steel bars very simple. The difficult part

* Corresponding author. Tel.: +38614768 613; FAX: +38614768629 
of the reinforced concrete section analysis is thus the integration of the stress field and the tangent material moduli of concrete.

A number of numerical methods has been proposed in order to make the integrations more efficient, see, e.g. Bonet et al. [2], Fafitis [8], Rasheed and Dinno [10]. The methods presented by Bonet et al. and Fafitis are particularly convenient when the stress field varies only in one direction. Their methods use Green's Theorem and transform the area integral into the boundary integral, which is then integrated numerically. While such an approach is more efficient than the one using directly the area integrals, it is still not computationally optimal due to the fact that the numerical integration inherently introduces errors. Moreover, the error in the cross-sectional integrals might imply a substantial error in force-deflection curves near the ultimate load (Saje et al. [12]). The error can, clearly, be made smaller by increasing the number of integration points, yet this increases the computational time and, consequently, reduces the time-efficiency of the overall finite-element algorithm. To make the integration procedure both time-efficient and exact, we have developed an analytical integration scheme as described in the sequel.

For the deduction of an analytical integration algorithm, the constitutive law of concrete needs to be prescribed in an analytical form. We have chosen the constitutive law proposed by Desayi and Krishnan [4] for the concrete in compression, and that of Bergan and Holand [1] for the concrete in tension. The next assumption concerns the strain distribution over the cross-section. We follow the standard approach in reinforced concrete beam analysis and assume the linear strain distribution (see, e.g. Brøndum-Nielsen [3], El-Metwally et al. [7], Izzuddin et al. [9], and Rodriguez and Aristizabal-Ochoa [11]). For the linear strain distribution, it is easy to find a constant strain and stress direction. With the help of some change of integration variables and by the use of Green's Theorem, we transform area integrals into the path integrals along the boundary of the cross-section of known, analytically integrable functions. If the cross-section, possibly hollow, can be approximated by a polygon (which is often the case in practice), an efficient formula for the analytical integration follows. We assume a monotonic increase in strains with the increase of a load, and thus disregard strain-reversals at any point of the cross-section. This assumption limits the applicability of the present procedure to the analyses of the ultimate limit capacity and the serviceability state of a frame structure.

The exactness of an analytical approach is obvious, while its computational efficiency might be doubtful if the final analytical expressions become very cumbersome. We demonstrate the efficiency of the present method via three numerical examples, in which we compare the accuracy and the required number of floating-point operations with several numerical integration-based methods. 


\section{Constitutive law of concrete}

Following Desayi and Krishnan [4] and Bergan and Holand [1], the uniaxial stress-strain relation for concrete is given by a function, which is smooth almost everywhere, except at a finite number of discrete points (Fig. 1):

$$
\sigma(\varepsilon)=\left\{\begin{array}{ll}
0 & \varepsilon \leq \varepsilon_{\mathrm{u}} \\
2 f_{\mathrm{m}}\left|\varepsilon_{1}\right| \frac{\varepsilon}{\varepsilon_{1}^{2}+\varepsilon^{2}} & \varepsilon_{\mathrm{u}}<\varepsilon \leq \varepsilon_{\mathrm{r}} \\
\frac{\sigma_{\mathrm{r}}}{\varepsilon_{\mathrm{r}}-\varepsilon_{\mathrm{m}}}\left(\varepsilon-\varepsilon_{\mathrm{m}}\right) & \varepsilon_{\mathrm{r}}<\varepsilon \leq \varepsilon_{\mathrm{m}} \\
0 & \varepsilon_{\mathrm{m}}<\varepsilon
\end{array} .\right.
$$

Here $f_{\mathrm{m}}$ is strength of concrete in compression $\left(f_{\mathrm{m}}=\left|\sigma_{\min }\right|>0\right) ; \varepsilon_{1}<0$ is strain at $f_{\mathrm{m}} ; \varepsilon_{\mathrm{u}}<0$ is ultimate strain in compression; $\varepsilon_{\mathrm{r}}>0$ is strain at tension strength of concrete, $\sigma_{\mathrm{r}}=2 f_{\mathrm{m}}\left|\varepsilon_{1}\right| \frac{\varepsilon_{\mathrm{r}}}{\varepsilon_{1}^{2}+\varepsilon_{\mathrm{r}}^{2}}$; and $\varepsilon_{\mathrm{m}}>0$ is ultimate strain in tension. Parameters $f_{\mathrm{m}}, \varepsilon_{1}$, and $\varepsilon_{\mathrm{u}}$ are determined in the compression tests on concrete cylinders; $\varepsilon_{\mathrm{r}}$ and $\varepsilon_{\mathrm{m}}$ must be determined in tension tests which are for concrete only rarely performed. The empirically proved and commonly used values $\varepsilon_{\mathrm{r}}=5.5 \cdot 10^{-5}$ and $\varepsilon_{\mathrm{m}}=7 \cdot 10^{-4}$ are rather good approximative values $[1]$.

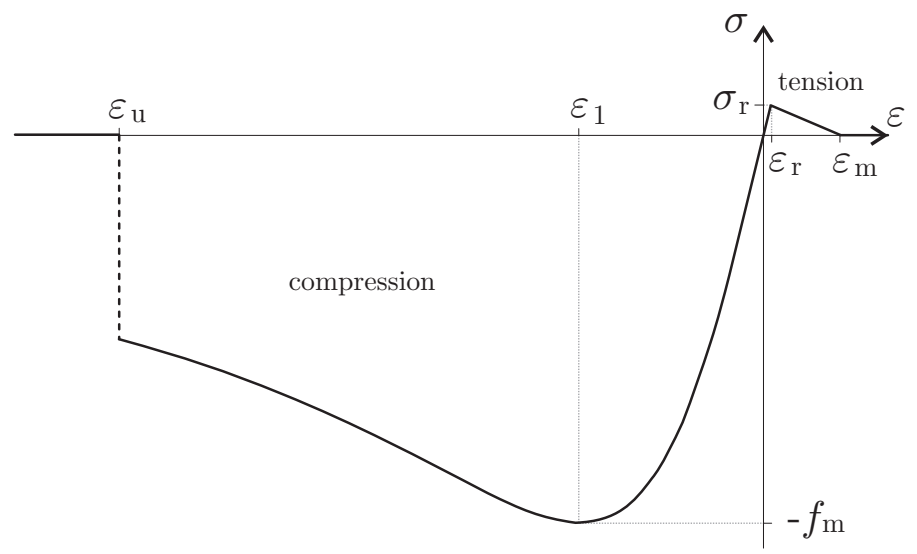

Fig. 1. Constitutive law of concrete. 


\section{Analytical cross-sectional integration}

\subsection{Strain distribution over the cross-section}

In spatial beam elements we usually assume the Bernoulli hypothesis that a cross-section suffers only rigid rotation during deformation. This implies that the normal strain (axial strain) is linearly distributed over the cross-section:

$$
\varepsilon(y, z)=\gamma_{1}+y \kappa_{3}+z \kappa_{2} .
$$

Here, $\varepsilon$ is the normal (axial) strain at fibre $(y, z)$ (see Fig. 2 for the definition of the cross-section and coordinate axes $y, z), \gamma_{1}$ is the normal strain, and $\kappa_{2}$ and $\kappa_{3}$ are the rotational strains (curvatures) about $y$ and $z$ axes, respectively, at the centroid axis of the cross-section, $y=z=0$.

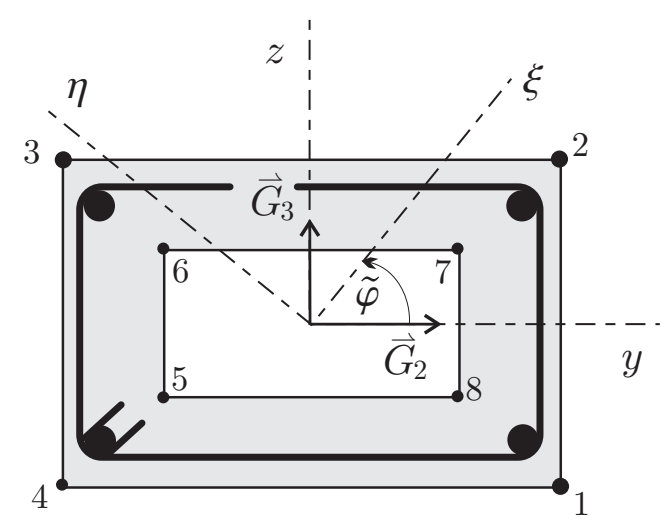

Fig. 2. Model of the cross-section and local coordinate systems.

The corresponding normal (axial) stress distribution over the cross-section, $\sigma(y, z)$, is determined from the constitutive law (1). The integration of stresses over the cross-sections yields axial force and bending moments with respect to the centroid of the cross-section:

$$
\begin{aligned}
N_{1} & =\iint_{\mathcal{A}} \sigma(\varepsilon(y, z)) d y d z \\
M_{2} & =\iint_{\mathcal{A}} z \sigma(\varepsilon(y, z)) d y d z \\
M_{3} & =\iint_{\mathcal{A}} y \sigma(\varepsilon(y, z)) d y d z .
\end{aligned}
$$




\subsection{Analytical integration of stresses}

We employ Green's Theorem and transform the area integrals (3) into path integrals along the cross-sectional boundary. We find it suitable to introduce a coordinate transformation first. Our goal is to introduce the rotated Cartesian coordinate system $(\xi, \eta)$, in which the stress field appears uniform in the $\eta$ direction: $\sigma(\xi, \eta)=\tilde{\sigma}(\eta)$. This can be achieved by a planar rotation of $(y, z)$ about $x$ for an angle $\tilde{\varphi}$ (see Fig. 2):

$$
\begin{aligned}
& \xi=y \cos \tilde{\varphi}+z \sin \tilde{\varphi} \\
& \eta=-y \sin \tilde{\varphi}+z \cos \tilde{\varphi}
\end{aligned}
$$

The normal strains with respect to $(\xi, \eta)$ are

$$
\begin{aligned}
\varepsilon(\xi, \eta) & =\gamma_{1}+(\xi \cos \tilde{\varphi}-\eta \sin \tilde{\varphi}) \kappa_{3}+(\xi \sin \tilde{\varphi}+\eta \cos \tilde{\varphi}) \kappa_{2} \\
& =\gamma_{1}+\xi\left(\kappa_{2} \sin \tilde{\varphi}+\kappa_{3} \cos \tilde{\varphi}\right)+\eta\left(\kappa_{2} \cos \tilde{\varphi}-\kappa_{3} \sin \tilde{\varphi}\right)
\end{aligned}
$$

Now we can choose such a rotation angle $\varphi$, that the coefficient at $\xi$ vanishes:

$$
\varphi=\operatorname{atan}\left(-\frac{\kappa_{3}}{\kappa_{2}}\right)
$$

Eq. (4) is singular at $\kappa_{2}=\kappa_{3}=0$. In this singular case, $\varepsilon(\xi, \eta)=\gamma_{1}$, which implies that the strain field is constant over the cross-section and can be moved from the integrals. In all the remaining cases, we may safely apply the coordinate transformation (4); thus

$$
\varepsilon=\gamma_{1}+K \eta, \quad K=\kappa_{2} \cos \varphi-\kappa_{3} \sin \varphi .
$$

Inserting Eq. (5) into Eq. (1) gives the normal stress as a function of coordinate $\eta$ only:

$$
\sigma(\varepsilon)=\hat{\sigma}\left(\gamma_{1}+K \eta\right)
$$

Eq. (5) relates $\varepsilon$ and $\eta$. Hence $\eta$ can be replaced by $\varepsilon$, and vice versa, whenever needed. Thus, a point $(\xi, \eta)$ can alternatively be identified by $(\xi, \varepsilon)$. This parametrization will be found useful in our integration procedure.

The application of the coordinate transformations in Eq. (3) yields

$$
\begin{aligned}
N_{1} & =\frac{1}{K} \iint_{\mathcal{A}} \sigma(\varepsilon) d \xi d \varepsilon \\
M_{2} & =\frac{1}{K} \iint_{\mathcal{A}}\left(\xi \sin \varphi+\frac{\varepsilon-\gamma_{1}}{K} \cos \varphi\right) \sigma(\varepsilon) d \xi d \varepsilon \\
M_{3} & =\frac{1}{K} \iint_{\mathcal{A}}\left(\xi \cos \varphi-\frac{\varepsilon-\gamma_{1}}{K} \sin \varphi\right) \sigma(\varepsilon) d \xi d \varepsilon
\end{aligned}
$$

where we have taken into account that the Jacobian of the coordinate transformation equals $\frac{1}{K}$. Green's Theorem makes it possible for the area integrals 
to be replaced by the path integrals along the boundary of the cross-section:

$$
\begin{aligned}
N_{1} & =\frac{1}{K} \int_{\partial \mathcal{A}} \xi \sigma(\varepsilon) d \varepsilon \\
M_{2} & =\frac{1}{K} \int_{\partial \mathcal{A}}\left(\frac{1}{2} \xi^{2} \sin \varphi+\xi \frac{\varepsilon-\gamma_{1}}{K} \cos \varphi\right) \sigma(\varepsilon) d \varepsilon \\
M_{3} & =\frac{1}{K} \int_{\partial \mathcal{A}}\left(\frac{1}{2} \xi^{2} \cos \varphi-\xi \frac{\varepsilon-\gamma_{1}}{K} \sin \varphi\right) \sigma(\varepsilon) d \varepsilon
\end{aligned}
$$

Remark 1 When employing the coordinate transformation in multiple integrals, the absolute value of the Jacobian determinant should be taken. The negative value of $K$ would have the same effect as changing the orientation of the boundary. We prescribe the orientation of the boundary by numbering the nodes in a counter-clockwise direction for the external boundary, and in a clockwise direction for the internal boundaries (see Fig. 2). The application of the coordinate transformation from $(y, z)$ to $(\xi, \varepsilon)$ changes the orientation of the boundary when $K$ becomes negative. This change is counteracted by the sign of $K$ in Eq. (6), which finally results in the proper sign of the integrals.

Let us now further assume that the cross-section can be approximated by a polygon (Fig. 2). For such a case, we can obtain an efficient formula for the analytical integration. The vertices of the cross-sectional boundary will be sorted in the positive direction of the external boundary, and in the negative direction of the internal boundaries describing the holes, both with respect to the coordinate system $(y, z)$ and then transformed to the coordinates $(\xi, \varepsilon)$ :

$$
\left(\xi_{\rho}, \varepsilon_{\rho}\right), \quad \rho=1, \ldots, N_{\rho} .
$$

$N_{\rho}$ denotes the number of vertices in the cross-section. The boundary between the successive vertices $\left(\xi_{\rho}, \varepsilon_{\rho}\right)$ and $\left(\xi_{\rho+1}, \varepsilon_{\rho+1}\right)$ is described by the linear function in the $(\xi, \varepsilon)$ coordinate system

$$
\xi(\varepsilon)=k_{\rho} \varepsilon+n_{\rho},
$$

where $k_{\rho}$ and $n_{\rho}$ are

$$
k_{\rho}=\frac{\xi_{\rho+1}-\xi_{\rho}}{\varepsilon_{\rho+1}-\varepsilon_{\rho}}, \quad n_{\rho}=\xi_{\rho}-k_{\rho} \varepsilon_{\rho} .
$$

With Eq. (7) the boundary integrals (6) can be decomposed into the sum of 
integrals between the successive vertices:

$$
\begin{aligned}
N_{1}=\frac{1}{K} \sum_{\rho=1}^{N_{\rho}} \int_{\varepsilon_{\rho}}^{\varepsilon_{\rho+1}} \xi \sigma(\varepsilon) d \varepsilon=\frac{1}{K} \sum_{\rho=1}^{N_{\rho}}\left[k_{\rho} \int_{\varepsilon_{\rho}}^{\varepsilon_{\rho+1}} \varepsilon \sigma(\varepsilon) d \varepsilon+n_{\rho} \int_{\varepsilon_{\rho}}^{\varepsilon_{\rho+1}} \sigma(\varepsilon) d \varepsilon\right] \\
M_{2}=\frac{1}{K} \sum_{\rho=1}^{N_{\rho}} \int_{\varepsilon_{\rho}}^{\varepsilon_{\rho+1}}\left(\frac{1}{2} \xi^{2} \sin \varphi+\xi \frac{\varepsilon-\gamma_{1}}{K} \cos \varphi\right) \sigma(\varepsilon) d \varepsilon \\
=\frac{1}{K} \sum_{\rho=1}^{N_{\rho}}\left[\left(\frac{k_{\rho}^{2}}{2} \sin \varphi+\frac{k_{\rho}}{K} \cos \varphi\right) \int_{\varepsilon_{\rho}}^{\varepsilon_{\rho+1}} \varepsilon^{2} \sigma(\varepsilon) d \varepsilon\right. \\
+\left(k_{\rho} n_{\rho} \sin \varphi+\frac{n_{\rho}-k_{\rho} \gamma_{1}}{K} \cos \varphi\right) \int_{\varepsilon_{\rho}}^{\varepsilon_{\rho+1}} \varepsilon \sigma(\varepsilon) d \varepsilon \\
\left.+\left(\frac{n_{\rho}^{2}}{2} \sin \varphi-\frac{n_{\rho} \gamma_{1}}{K} \cos \varphi\right) \int_{\varepsilon_{\rho}}^{\varepsilon_{\rho+1}} \sigma(\varepsilon) d \varepsilon\right] .
\end{aligned}
$$

An analogous procedure is employed for $M_{3}$, and a similar result is obtained, with the only difference being that coefficients $\sin \varphi$ and $\cos \varphi$ are replaced by $\cos \varphi$ and $-\sin \varphi$. Thus, it is suitable and numerically more efficient to prepare the coefficients for $M_{2}$ in $M_{3}$ simultaneously:

$$
\left[\begin{array}{ccc}
c_{\rho 12} & c_{\rho 22} & c_{\rho 32} \\
c_{\rho 13} & c_{\rho 23} & c_{\rho 33}
\end{array}\right]=\left[\begin{array}{cc}
\cos \varphi & \sin \varphi \\
-\sin \varphi & \cos \varphi
\end{array}\right]\left[\begin{array}{ccc}
\frac{k_{\rho}}{K} & \frac{n_{\rho}-k_{\rho} \gamma_{1}}{K} & -\frac{n_{\rho} \gamma_{1}}{K} \\
\frac{k_{\rho}^{2}}{2} & k_{\rho} n_{\rho} & \frac{n_{\rho}^{2}}{2}
\end{array}\right] .
$$

Then we can write

$M_{j}=\frac{1}{K} \sum_{\rho=1}^{N_{\rho}}\left[c_{\rho 1 j} \int_{\varepsilon_{\rho}}^{\varepsilon_{\rho+1}} \varepsilon^{2} \sigma(\varepsilon) d \varepsilon+c_{\rho 2 j} \int_{\varepsilon_{\rho}}^{\varepsilon_{\rho+1}} \varepsilon \sigma(\varepsilon) d \varepsilon+c_{\rho 3 j} \int_{\varepsilon_{\rho}}^{\varepsilon_{\rho+1}} \sigma(\varepsilon) d \varepsilon\right], j=2,3$.

We can replace the definite integrals $\int_{\varepsilon_{\rho}}^{\varepsilon_{\rho+1}} \sigma(\varepsilon) d \varepsilon, \int_{\varepsilon_{\rho}}^{\varepsilon_{\rho+1}} \varepsilon \sigma(\varepsilon) d \varepsilon$, and $\int_{\varepsilon_{\rho}}^{\varepsilon_{\rho+1}} \varepsilon^{2} \sigma(\varepsilon) d \varepsilon$ by analytical expressions $I_{1 \rho}, I_{2 \rho}$, and $I_{3 \rho}$ as soon as we employ the proposed constitutive law (1) in an analytical form. Thus

$$
\begin{aligned}
& N_{1}=\frac{1}{K} \sum_{\rho=1}^{N_{\rho}}\left(k_{\rho} I_{2 \rho}+n_{\rho} I_{1 \rho}\right) \\
& M_{j}=\frac{1}{K} \sum_{\rho=1}^{N_{\rho}}\left(c_{\rho 1 j} I_{3 \rho}+c_{\rho 2 j} I_{2 \rho}+c_{\rho 3 j} I_{1 \rho}\right), \quad j=2,3 .
\end{aligned}
$$

In Eq. (8) we must take a special care to pin-point the discrete points at which the first derivative of the function $\sigma(\varepsilon)$ does not exist. For the part of the cross-section, $\mathcal{A}_{u}$, where the condition $\varepsilon_{\mathrm{u}} \leq \varepsilon \leq \varepsilon_{\mathrm{r}}$ is met, the expressions 
$I_{1 \rho}, I_{2 \rho}$ and $I_{3 \rho}$ are determined by

$$
\begin{aligned}
& I_{1 \rho}=f_{\mathrm{m}}\left|\varepsilon_{1}\right| \ln \frac{\varepsilon_{1}^{2}+\varepsilon_{\rho+1}^{2}}{\varepsilon_{1}^{2}+\varepsilon_{\rho}^{2}} \\
& I_{2 \rho}=2 f_{\mathrm{m}}\left|\varepsilon_{1}\right|\left(\varepsilon_{\rho+1}-\varepsilon_{\rho}+\varepsilon_{1}\left(\operatorname{atan} \frac{\varepsilon_{\rho}}{\varepsilon_{1}}-\operatorname{atan} \frac{\varepsilon_{\rho+1}}{\varepsilon_{1}}\right)\right) \\
& I_{3 \rho}=f_{\mathrm{m}}\left|\varepsilon_{1}\right|\left(\varepsilon_{\rho+1}^{2}-\varepsilon_{\rho}^{2}+\varepsilon_{1}^{2} \ln \frac{\varepsilon_{1}^{2}+\varepsilon_{\rho}^{2}}{\varepsilon_{1}^{2}+\varepsilon_{\rho+1}^{2}}\right) .
\end{aligned}
$$

For the part of the cross-section, $\mathcal{A}_{m}$, where $\varepsilon_{\mathrm{r}} \leq \varepsilon \leq \varepsilon_{\mathrm{m}}$ holds, we establish $I_{1 \rho}, I_{2 \rho}$ and $I_{3 \rho}$ by the formulae

$$
\begin{aligned}
& I_{1 \rho}=\frac{\sigma_{\mathrm{r}}}{\varepsilon_{\mathrm{r}}-\varepsilon_{\mathrm{m}}}\left(\frac{1}{2}\left(\varepsilon_{\rho+1}^{2}-\varepsilon_{\rho}^{2}\right)-\varepsilon_{\mathrm{m}}\left(\varepsilon_{\rho+1}-\varepsilon_{\rho}\right)\right) \\
& I_{2 \rho}=\frac{\sigma_{\mathrm{r}}}{\varepsilon_{\mathrm{r}}-\varepsilon_{\mathrm{m}}}\left(\frac{1}{3}\left(\varepsilon_{\rho+1}^{3}-\varepsilon_{\rho}^{3}\right)-\frac{\varepsilon_{\mathrm{m}}}{2}\left(\varepsilon_{\rho+1}^{2}-\varepsilon_{\rho}^{2}\right)\right) \\
& I_{3 \rho}=\frac{\sigma_{\mathrm{r}}}{\varepsilon_{\mathrm{r}}-\varepsilon_{\mathrm{m}}}\left(\frac{1}{4}\left(\varepsilon_{\rho+1}^{4}-\varepsilon_{\rho}^{4}\right)-\frac{\varepsilon_{\mathrm{m}}}{3}\left(\varepsilon_{\rho+1}^{3}-\varepsilon_{\rho}^{3}\right)\right) .
\end{aligned}
$$

\subsection{Analytical integration of tangent material moduli}

For the iterative solution of the governing equations of the beam, we also need to calculate the tangent constitutive matrix of the cross-section. This requires various integrals of tangent material moduli over the cross-section to be evaluated. We differentiate Eqs. (3) with respect to the axial strain and the curvatures:

$$
\begin{aligned}
\frac{\partial N_{1}}{\partial \gamma_{1}} & =\iint_{\mathcal{A}} \frac{d \sigma}{d \varepsilon} d y d z \\
\frac{\partial N_{1}}{\partial \kappa_{2}} & =\frac{\partial M_{2}}{\partial \gamma_{1}}=\iint_{\mathcal{A}} z \frac{d \sigma}{d \varepsilon} d y d z \\
\frac{\partial N_{1}}{\partial \kappa_{3}} & =\frac{\partial M_{3}}{\partial \gamma_{1}}=\iint_{\mathcal{A}} y \frac{d \sigma}{d \varepsilon} d y d z \\
\frac{\partial M_{2}}{\partial \kappa_{2}} & =\iint_{\mathcal{A}} z^{2} \frac{d \sigma}{d \varepsilon} d y d z \\
\frac{\partial M_{2}}{\partial \kappa_{3}} & =\frac{\partial M_{3}}{\partial \kappa_{2}}=\iint_{\mathcal{A}} y z \frac{d \sigma}{d \varepsilon} d y d z \\
\frac{\partial M_{3}}{\partial \kappa_{3}} & =\iint_{\mathcal{A}} y^{2} \frac{d \sigma}{d \varepsilon} d y d z
\end{aligned}
$$

The integration scheme (8) holds also for (11), only that the analytical expressions for the definite integrals of $\frac{d \sigma}{d \varepsilon}$, $\varepsilon \frac{d \sigma}{d \varepsilon}$, and $\varepsilon^{2} \frac{d \sigma}{d \varepsilon}$ differ. An analogous 
procedure to the one given in Section 3.2 can therefore be applied in integrating (11) and (12). Only the derivation of the integration scheme for $\frac{\partial M_{2}}{\partial \kappa_{2}}$ will be shown in detail. By the use of coordinate transformations (4)-(5) and upon applying Green's Theorem, we get

$$
\begin{aligned}
\frac{\partial M_{2}}{\partial \kappa_{2}}=\frac{1}{K} \int_{\partial \mathcal{A}} & \left(\frac{1}{3} \xi^{3} \cos ^{2} \varphi-\frac{1}{K} \varepsilon \xi^{2} \sin \varphi+\frac{1}{K^{2}} \varepsilon^{2} \xi \sin ^{2} \varphi\right. \\
+ & \left.\frac{1}{K} \gamma_{1} \xi^{2} \sin \varphi \cos \varphi-\frac{2 \gamma_{1}}{K^{2}} \varepsilon \xi \sin ^{2} \varphi+\frac{\gamma_{1}^{2}}{K^{2}} \xi \sin ^{2} \varphi\right) \sigma^{\prime}(\varepsilon) d \varepsilon
\end{aligned}
$$

Assuming once again a polygonal boundary of the cross-section and employing Eq. (7), we obtain

$$
\begin{aligned}
\frac{\partial M_{2}}{\partial \kappa_{2}}=\frac{1}{K} \sum_{\rho=1}^{N_{\rho}} & {\left[d_{\rho 1} \int_{\varepsilon_{\rho}}^{\varepsilon_{\rho+1}} \varepsilon^{3} \sigma(\varepsilon) d \varepsilon+d_{\rho 2} \int_{\varepsilon_{\rho}}^{\varepsilon_{\rho+1}} \varepsilon^{2} \sigma(\varepsilon) d \varepsilon\right.} \\
& \left.+d_{\rho 3} \int_{\varepsilon_{\rho}}^{\varepsilon_{\rho+1}} \varepsilon \sigma(\varepsilon) d \varepsilon+d_{\rho 4} \int_{\varepsilon_{\rho}}^{\varepsilon_{\rho+1}} \sigma(\varepsilon) d \varepsilon\right]
\end{aligned}
$$

where the coefficients $d_{\rho}$ are

$$
\begin{aligned}
& d_{\rho 1}=\frac{k_{\rho}^{3}}{3} \sin ^{2} \varphi+\frac{k_{\rho}^{2}}{K} \sin \varphi \cos \varphi+\frac{k_{\rho}}{K^{2}} \cos ^{2} \varphi \\
& d_{\rho 2}=k_{\rho}^{2} n_{\rho} \sin ^{2} \varphi+\frac{2 k_{\rho} n_{\rho}-k_{\rho}^{2} \gamma_{1}}{K} \sin \varphi \cos \varphi+\frac{n_{\rho}-2 k_{\rho} \gamma_{1}}{K^{2}} \cos ^{2} \varphi \\
& d_{\rho 3}=k_{\rho} n_{\rho}^{2} \sin ^{2} \varphi+\frac{n_{\rho}^{2}-2 k_{\rho} n_{\rho} \gamma_{1}}{K} \sin \varphi \cos \varphi+\frac{k_{\rho} \gamma^{2}-2 n_{\rho} \gamma_{1}}{K^{2}} \cos ^{2} \varphi \\
& d_{\rho 4}=\frac{n_{\rho}^{3}}{3} \sin ^{2} \varphi-\frac{n_{\rho}^{2} \gamma_{1}}{K} \sin \varphi \cos \varphi+\frac{n_{\rho} \gamma_{1}^{2}}{K^{2}} \cos ^{2} \varphi .
\end{aligned}
$$

The remaining integrals in (12) are tackled similarly. Therefore, we can define a general matrix form for defining the coefficients $d_{\rho}$

$$
\left[\begin{array}{cccc}
d_{\rho 11} & d_{\rho 21} & d_{\rho 31} & d_{\rho 41} \\
d_{\rho 12} & d_{\rho 22} & d_{\rho 32} & d_{\rho 42} \\
d_{\rho 13} & d_{\rho 23} & d_{\rho 33} & d_{\rho 43}
\end{array}\right]=\mathbf{Q}\left[\begin{array}{cccc}
\frac{k_{\rho}^{3}}{3} & k_{\rho}^{2} n_{\rho} & k_{\rho} n_{\rho}^{2} & \frac{n_{\rho}^{3}}{3} \\
\frac{k_{\rho}^{2}}{K} & \frac{2 k_{\rho} n_{\rho}-k_{\rho}^{2} \gamma_{1}}{K} & \frac{n_{\rho}^{2}-2 k_{\rho} n_{\rho} \gamma_{1}}{K} & -\frac{n_{\rho}^{2} \gamma_{1}}{K} \\
\frac{k_{\rho}}{K^{2}} & \frac{n_{\rho}-2 k_{\rho} \gamma_{1}}{K^{2}} & \frac{k_{\rho} \gamma^{2}-2 n_{\rho} \gamma_{1}}{K^{2}} & \frac{n_{\rho} \gamma_{1}^{2}}{K^{2}}
\end{array}\right],
$$

where

$$
\mathbf{Q}=\left[\begin{array}{ccc}
\sin ^{2} \varphi & \sin \varphi \cos \varphi & \cos ^{2} \varphi \\
\sin \varphi \cos \varphi & \frac{1}{2}\left(\cos ^{2} \varphi-\sin ^{2} \varphi\right) & -\sin \varphi \cos \varphi \\
\cos ^{2} \varphi & -\sin \varphi \cos \varphi & \sin ^{2} \varphi
\end{array}\right]
$$


The integrals (12) can then be expressed with a general formula

$$
\begin{aligned}
\frac{\partial M_{i}}{\partial \kappa_{j}}=\frac{1}{K} \sum_{\rho=1}^{N_{\rho}} & {\left[d_{\rho 1, i+j-3} \int_{\varepsilon_{\rho}}^{\varepsilon_{\rho+1}} \varepsilon^{3} \sigma(\varepsilon) d \varepsilon+d_{\rho 2, i+j-3} \int_{\varepsilon_{\rho}}^{\varepsilon_{\rho+1}} \varepsilon^{2} \sigma(\varepsilon) d \varepsilon\right.} \\
& \left.+d_{\rho 3, i+j-3} \int_{\varepsilon_{\rho}}^{\varepsilon_{\rho+1}} \varepsilon \sigma(\varepsilon) d \varepsilon+d_{\rho 4, i+j-3} \int_{\varepsilon_{\rho}}^{\varepsilon_{\rho+1}} \sigma(\varepsilon) d \varepsilon\right], \quad i, j=2,3 .
\end{aligned}
$$

For convenience, the integration scheme for the tangent material moduli of the cross-section are summarized below:

$$
\begin{aligned}
& \frac{\partial N_{1}}{\partial \gamma_{1}}=\frac{1}{K} \sum_{\rho=1}^{N_{\rho}}\left(k_{\rho} I_{2 \rho}^{\prime}+n_{\rho} I_{1 \rho}^{\prime}\right) \\
& \frac{\partial N_{1}}{\partial \kappa_{j}}=\frac{1}{K} \sum_{\rho=1}^{N_{\rho}}\left(c_{\rho 1 j} I_{3 \rho}^{\prime}+c_{\rho 2 j} I_{2 \rho}^{\prime}+c_{\rho 3 j} I_{1 \rho}^{\prime}\right) \\
& \frac{\partial M_{i}}{\partial \kappa_{j}}=\frac{1}{K} \sum_{\rho=1}^{N_{\rho}}\left(d_{\rho 1, i+j-3} I_{4 \rho}^{\prime}+d_{\rho 2, i+j-3} I_{3 \rho}^{\prime}+d_{\rho 3, i+j-3} I_{2 \rho}^{\prime}+d_{\rho 4, i+j-3} I_{1 \rho}^{\prime}\right)
\end{aligned}
$$

Subregion $\mathcal{A}_{u}: \varepsilon_{\mathrm{u}} \leq \varepsilon \leq \varepsilon_{\mathrm{r}}$

$$
\begin{aligned}
& I_{1 \rho}^{\prime}=2 f_{\mathrm{m}}\left|\varepsilon_{1}\right|\left(\frac{\varepsilon_{\rho+1}}{\varepsilon_{1}^{2}+\varepsilon_{\rho+1}^{2}}-\frac{\varepsilon_{\rho}}{\varepsilon_{1}^{2}+\varepsilon_{\rho}^{2}}\right) \\
& I_{2 \rho}^{\prime}=2 f_{\mathrm{m}}\left|\varepsilon_{1}\right|^{3}\left(\frac{1}{\varepsilon_{1}^{2}+\varepsilon_{\rho}^{2}}-\frac{1}{\varepsilon_{1}^{2}+\varepsilon_{\rho+1}^{2}}\right)+f_{\mathrm{m}}\left|\varepsilon_{1}\right| \ln \frac{\varepsilon_{1}^{2}+\varepsilon_{\rho}^{2}}{\varepsilon_{1}^{2}+\varepsilon_{\rho+1}^{2}} \\
& I_{3 \rho}^{\prime}=2 f_{\mathrm{m}}\left|\varepsilon_{1}\right|\left(\varepsilon_{\rho}-\varepsilon_{\rho+1}\right)-\varepsilon_{1}^{2} I_{1 \rho}^{\prime}+4 f_{\mathrm{m}} \varepsilon_{1}^{2}\left(\operatorname{atan} \frac{\varepsilon_{\rho+1}}{\varepsilon_{1}}-\operatorname{atan} \frac{\varepsilon_{\rho}}{\varepsilon_{1}}\right) \\
& I_{4 \rho}^{\prime}=f_{\mathrm{m}}\left|\varepsilon_{1}\right|\left(\varepsilon_{\rho}^{2}-\varepsilon_{\rho+1}^{2}\right)+2 f_{\mathrm{m}}\left|\varepsilon_{1}\right|^{5}\left(\frac{1}{\varepsilon_{1}^{2}+\varepsilon_{\rho}^{2}}-\frac{1}{\varepsilon_{1}^{2}+\varepsilon_{\rho+1}^{2}}\right) \\
& \quad+3 f_{\mathrm{m}}\left|\varepsilon_{1}\right|^{3} \ln \frac{\varepsilon_{1}^{2}+\varepsilon_{\rho+1}^{2}}{\varepsilon_{1}^{2}+\varepsilon_{\rho}^{2}}
\end{aligned}
$$

Subregion $\mathcal{A}_{m}: \varepsilon_{\mathrm{r}} \leq \varepsilon \leq \varepsilon_{\mathrm{m}}$

$$
\begin{aligned}
I_{1 \rho}^{\prime} & =\frac{\sigma_{\mathrm{r}}}{\varepsilon_{\mathrm{r}}-\varepsilon_{\mathrm{m}}}\left(\varepsilon_{\rho+1}-\varepsilon_{\rho}\right) \\
I_{2 \rho}^{\prime} & =\frac{1}{2} \frac{\sigma_{\mathrm{r}}}{\varepsilon_{\mathrm{r}}-\varepsilon_{\mathrm{m}}}\left(\varepsilon_{\rho+1}^{2}-\varepsilon_{\rho}^{2}\right) \\
I_{3 \rho}^{\prime} & =\frac{1}{3} \frac{\sigma_{\mathrm{r}}}{\varepsilon_{\mathrm{r}}-\varepsilon_{\mathrm{m}}}\left(\varepsilon_{\rho+1}^{3}-\varepsilon_{\rho}^{3}\right) \\
I_{4 \rho}^{\prime} & =\frac{1}{4} \frac{\sigma_{\mathrm{r}}}{\varepsilon_{\mathrm{r}}-\varepsilon_{\mathrm{m}}}\left(\varepsilon_{\rho+1}^{4}-\varepsilon_{\rho}^{4}\right) .
\end{aligned}
$$




\section{Numerical integration methods}

In this section we briefly describe three different numerical methods which will be used in the next section in comparisons of the present method with some other approaches. It is true that analytical methods give exact values, but they are often neglected in practice when they require relatively large computational times compared to a numerical method. Thus it is also important to compare the computational times of analytical and numerical approaches.

We will compare the following three numerical integration-based methods, using

i) the numerical path integration along the boundary;

ii) the numerical integration over thin layers of the cross-section;

iii) the 2-dimensional numerical integration over the cross-section.

\subsection{Numerical path integration along the boundary}

The method is essentially analogous to the one developed by Bonet et al. [2], the difference being only in the different parametrization, here defined by Eqs. (4) and (5). The integrals $I_{1 \rho}, I_{2 \rho}, I_{3 \rho}, I_{1 \rho}^{\prime}, I_{2 \rho}^{\prime}, I_{3 \rho}^{\prime}$ and $I_{4 \rho}^{\prime}$ over the boundaries of the subregions $\mathcal{A}_{u}$ and $\mathcal{A}_{m}$ are now not evaluated analytically but numerically by Gaussian integration between the successive vertices. So, the integrations are substituted by the weighted sums

$$
\int_{\varepsilon_{\rho}}^{\varepsilon_{\rho+1}} f(\varepsilon) d \varepsilon \quad \rightarrow \quad \frac{\varepsilon_{\rho+1}-\varepsilon_{\rho}}{2} \sum_{p=1}^{n} w_{p} f\left(\varepsilon_{p}\right),
$$

where $w_{p}$ are the integration weights and $\varepsilon_{p}$ are the integration points.

After applying (13) we obtain the following formulae:

$$
\begin{aligned}
I_{1 \rho} & =\frac{\varepsilon_{\rho+1}-\varepsilon_{\rho}}{2} \sum_{p=1}^{n} w_{p} \sigma\left(\varepsilon_{p}\right) \\
I_{2 \rho} & =\frac{\varepsilon_{\rho+1}-\varepsilon_{\rho}}{2} \sum_{p=1}^{n} w_{p} \varepsilon_{p} \sigma\left(\varepsilon_{p}\right) \\
I_{3 \rho} & =\frac{\varepsilon_{\rho+1}-\varepsilon_{\rho}}{2} \sum_{p=1}^{n} w_{p} \varepsilon_{p}^{2} \sigma\left(\varepsilon_{p}\right) \\
I_{i \rho}^{\prime} & =\frac{\varepsilon_{\rho+1}-\varepsilon_{\rho}}{2} \sum_{p=1}^{n} w_{p} \varepsilon_{p}^{i-1} \sigma^{\prime}\left(\varepsilon_{p}\right), \quad i=1,2,3,4,
\end{aligned}
$$

where function $\sigma(\varepsilon)=2 f_{\mathrm{m}}\left|\varepsilon_{1}\right| \frac{\varepsilon}{\varepsilon_{1}^{2}+\varepsilon^{2}}$ is taken on subregion $\mathcal{A}_{u}$, and $\sigma(\varepsilon)=$ 
$\frac{\sigma_{\mathrm{r}}}{\varepsilon_{\mathrm{r}}-\varepsilon_{\mathrm{m}}}\left(\varepsilon-\varepsilon_{\mathrm{m}}\right)$ on $\mathcal{A}_{m}$.

\subsection{Numerical integration over thin layers}

This method divides the integration region into layers and executes numerically the area integrals over the layers. The Cartesian coordinates $(\xi, \eta)$ are used. The stress field then appears uniform in $\eta$ direction: $\sigma=\hat{\sigma}\left(\gamma_{1}+K \eta\right)$ (see Eq. (4)). The additional coordinate transformation (5) is not necessary for this method. The uniform behaviour of stress field in one direction simplifies the area integrals considerably, if the boundaries in the $\xi$ direction are independent on $\eta$. For such a simplified case we can write

$$
\iint_{\mathcal{A}} \sigma(\varepsilon(y, z)) d y d z=\int_{\xi_{1}}^{\xi_{2}} \int_{\eta_{1}}^{\eta_{2}} \sigma d \xi d \eta=\left(\xi_{2}-\xi_{1}\right) \int_{\eta_{1}}^{\eta_{2}} \sigma d \eta
$$

Unfortunately, this is very rarely the case, so that in general, we have to divide the integration area into thin layers. Prior to the division, however, we find it suitable to determine the subregions $\mathcal{A}_{u}$, in which the condition $\varepsilon_{\mathrm{u}} \leq \frac{\eta-\gamma_{1}}{K} \leq \varepsilon_{\mathrm{r}}$ is met, and $\mathcal{A}_{m}$, where $\varepsilon_{\mathrm{r}} \leq \frac{\eta-\gamma_{1}}{K} \leq \varepsilon_{\mathrm{m}}$ holds. $\mathcal{A}_{u}$ and $\mathcal{A}_{m}$, are bounded by the minimum and maximum values of coordinate $\eta: \eta_{\min }$ and $\eta_{\text {max }}$. The interval $\left[\eta_{\min }, \eta_{\max }\right]$ is then divided into $l$ layers $\left[\eta_{s}, \eta_{s+1}\right]$, where $\eta_{1}=\eta_{\min }$ and $\eta_{l+1}=\eta_{\max }$. In a layer, the boundaries in the $\xi$ direction are approximated by their values at the midpoint $\eta=\frac{\eta_{s}+\eta_{s+1}}{2}$, so that the layers are modelled by the rectangles. Thus we have

$$
\begin{aligned}
& N_{1}=\sum_{s=1}^{l}\left(\xi_{s 2}-\xi_{s 1}\right) \int_{\eta_{s}}^{\eta_{s+1}} \sigma d \eta \\
& M_{2}=\sum_{s=1}^{l}\left(\xi_{s 2}-\xi_{s 1}\right) \cos \varphi \int_{\eta_{s}}^{\eta_{s+1}} \eta \sigma d \eta+\sum_{s=1}^{l} \frac{1}{2}\left(\xi_{s 2}^{2}-\xi_{s 1}^{2}\right) \sin \varphi \int_{\eta_{s}}^{\eta_{s+1}} \sigma d \eta \\
& M_{3}=-\sum_{s=1}^{l}\left(\xi_{s 2}-\xi_{s 1}\right) \sin \varphi \int_{\eta_{s}}^{\eta_{s+1}} \eta \sigma d \eta+\sum_{s=1}^{l} \frac{1}{2}\left(\xi_{s 2}^{2}-\xi_{s 1}^{2}\right) \cos \varphi \int_{\eta_{s}}^{\eta_{s+1}} \sigma d \eta .
\end{aligned}
$$

The integrals over the $\eta$-direction are evaluated numerically by the one-dimensional Gaussian integration. Similar procedure is used for the integrations of the tangent material moduli over the cross-section.

As the subregions $\mathcal{A}_{u}$ and $\mathcal{A}_{m}$ are analyzed separately, we can use different number of layers over each subregion. E.g., in $\mathcal{A}_{m}$ a much smaller number of layers is needed compared to $\mathcal{A}_{u}$ as the tension part of the concrete crosssection is usually relatively small.

Furthermore, the optimisation of the number and the thicknesses of layers would be possible in order to enhance the efficiency of the method; we will 
not proceed in this direction, however, because our primary concern is the analytical integration.

Remark 2 Note that the number of boundary values in the $\xi$-direction might be larger than two for hollow or non-convex cross-sections. In such cases, the layer is further divided into sublayers in the $\xi$-direction and the numerical integration over each sublayer is performed.

\subsection{Numerical 2-dimensional integration}

The last method is the simplest, but the most general. The area integrals are evaluated by the use of a double Gaussian integration:

$$
\int_{\xi_{1}}^{\xi_{2}} \int_{\eta_{1}}^{\eta_{2}} f(\xi, \eta) d \xi d \eta=\frac{\xi_{2}-\xi_{1}}{2} \frac{\eta_{2}-\eta_{1}}{2} \sum_{p=1}^{n} \sum_{q=1}^{m} w_{p} w_{q} f\left(\xi_{p}, \eta_{q}\right)
$$

$w_{p}$ and $w_{q}$ are the weights of Gaussian integration, and $\left(\xi_{p}, \eta_{q}\right)$ are the integration points.

If the shape of the integration area is not rectangular (a rectangle was in fact tacitly assumed in Eq. (14)), we can still use the same method by the following generalization. The smallest rectangle is found in which the whole integration area lies. Eq. (14) is then applied over this rectangle, taking the values $f\left(\xi_{p}, \eta_{q}\right)$ in integration points outside the cross-section to be zero.

In order to make fair comparisons of this numerical method to the previous ones, we further improve its accuracy by using the coordinate transformation and determine tension and compression subregions as in the method of Section 4.2. Thus, we first determine $\mathcal{A}_{u}$ and $\mathcal{A}_{m}$, find their smallest rectangular areas, set the values of stresses and their derivatives outside the section to zero, and only then employ Eq. (14) to evaluate integrals (3), (11) and (12).

\section{$5 \quad$ Numerical examples}

An analytical approach is preferable, when we need the exact values. This is usually not the case in the finite-element simulations, where several different sources of error emerge and exact values of intermediate variables do not improve the overall solution's accuracy. When so, we tend to choose less accurate numerically obtained approximative values, if they can be evaluated more rapidly than the analytical ones. This does not hold for the present analytical integration routine, as we will show by numerical tests. 
For the evaluation of results of various methods, two measures will be used: (i) the relative error (in per cent) with respect to the exact value, and (ii) the number of floating-point operations, flops, needed to obtain the results. The stress resultants and the six components of the tangent constitutive matrix of the cross-section are obtained simultaneously in order to avoid the repetitions of some calculations. The algorithms were implemented and tested in Matlab [6].

Different types of cross-sections have been analyzed. Here we present three cross-sections that are interesting because of their non-trivial shape. For all the cases the following parameters of concrete are taken:

$$
\begin{aligned}
\varepsilon_{\mathrm{u}} & =-8 \cdot 10^{-3} \\
\varepsilon_{1} & =-2.2 \cdot 10^{-3} \\
\varepsilon_{\mathrm{r}} & =5.5 \cdot 10^{-5} \\
\varepsilon_{\mathrm{m}} & =7 \cdot 10^{-4} \\
f_{\mathrm{m}} & =3.3 \mathrm{kN} / \mathrm{cm}^{2} .
\end{aligned}
$$

The geometry of the cross-section will be given in each case separately.

The analytical integration scheme developed above has been successfully incorporated into the strain-based spatial beam finite-element formulation of Zupan and Saje [13]. The formulation employs the strain vectors as the only interpolated variables and is thus particularly advantageous in materially nonlinear problems.

\section{1 "I-shaped" cross-section}

Our first numerical example is an "I-shaped" cross-section, shown in Fig. 3a. The cross-section is made up of plain concrete with no reinforcement. Such a cross-section seems to be a severe test of the method. The integrals were evaluated for the linear strain distribution, described by the strain resultants:

$$
\gamma_{1}=-0.0010, \quad \kappa_{2}=-0.0002, \quad \kappa_{3}=0.0001
$$

The coordinate transformation and the subregion determination are made first. The result is shown in Fig. 3b. Note, however, that for the two methods, which use the area integrals, the scale on the $\varepsilon$ axis is different because the rescaling in the $\eta$ direction is omitted.

The exact results for the axial force, the bending moments and the crosssectional constitutive matrix are shown in Table 1 and compared to the results of numerically integrated path integrals. The results for the stress-resultants, obtained by 2-point Gaussian integration over the boundary, compare well, the largest relative error being less than $4 \%$. The error of the 2-point Gaussian integration in the cross-sectional tangent constitutive matrix is, on the other 


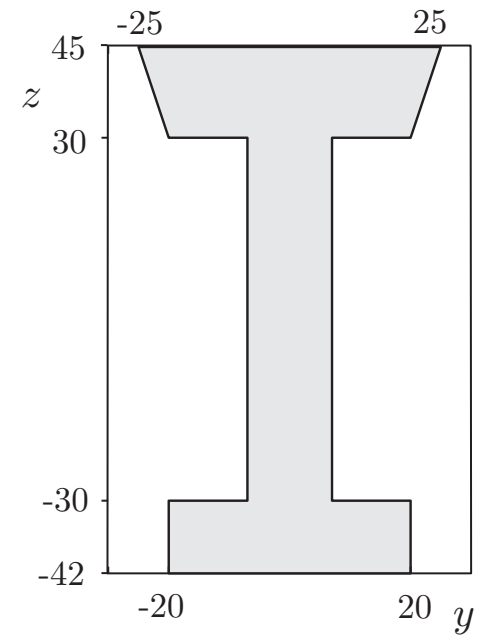

a)

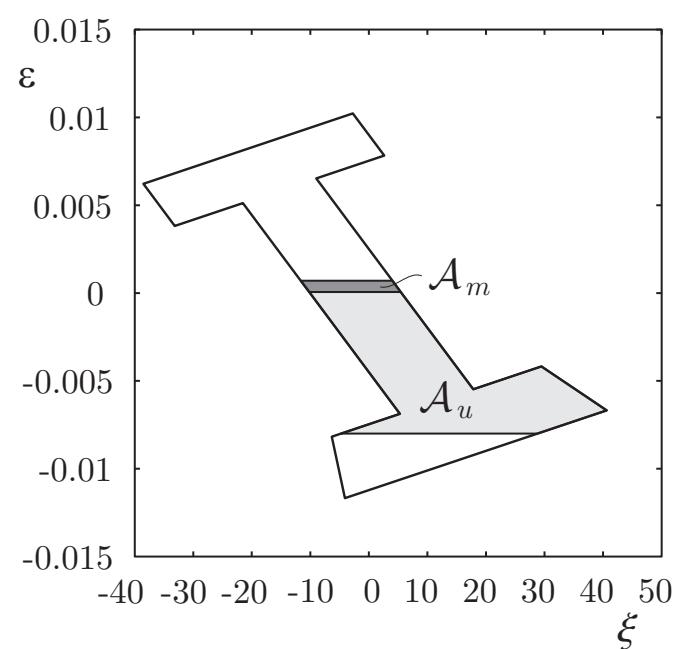

b)

Fig. 3. a) Geometry of the "I-shaped" section and b) its image in coordinate system $(\xi, \varepsilon)$ with subregions $\mathcal{A}_{u}$ and $\mathcal{A}_{m}$.

Table 1

Analytical results for the cross-section in Fig. 3 (left) and relative error (in per cent) of the numerical results based on Gaussian path integral along the boundary (right)

\begin{tabular}{|c|r|}
\hline quantity & \multicolumn{1}{c|}{ value } \\
\hline$N_{1}$ & $-1.88 \cdot 10^{3}$ \\
$M_{2}$ & $-3.67 \cdot 10^{4}$ \\
$M_{3}$ & $-6.58 \cdot 10^{3}$ \\
$\partial N_{1} / \partial \gamma_{1}$ & $5.25 \cdot 10^{4}$ \\
$\partial N_{1} / \partial \kappa_{2}$ & $-4.72 \cdot 10^{6}$ \\
$\partial N_{1} / \partial \kappa_{3}$ & $-8.38 \cdot 10^{5}$ \\
$\partial M_{2} / \partial \kappa_{2}$ & $-1.19 \cdot 10^{8}$ \\
$\partial M_{2} / \partial \kappa_{3}$ & $-2.73 \cdot 10^{7}$ \\
$\partial M_{3} / \partial \kappa_{3}$ & $-1.38 \cdot 10^{7}$ \\
\hline flops & 2597 \\
\hline
\end{tabular}

\begin{tabular}{|c|rrrr|}
\hline & $n=2$ & $n=3$ & $n=5$ & $n=10$ \\
\hline$N_{1}$ & 3.4 & 0.3 & 0.01 & 0.00 \\
$M_{2}$ & 2.1 & 0.3 & 0.01 & 0.00 \\
$M_{3}$ & 1.6 & 0.2 & 0.01 & 0.00 \\
$\partial N_{1} / \partial \gamma_{1}$ & 0.2 & $\mathbf{4 3 . 9}$ & 2.26 & 0.00 \\
$\partial N_{1} / \partial \kappa_{2}$ & $\mathbf{1 5 . 4}$ & 1.9 & 0.07 & 0.00 \\
$\partial N_{1} / \partial \kappa_{3}$ & 8.3 & 5.0 & 0.41 & 0.00 \\
$\partial M_{2} / \partial \kappa_{2}$ & 3.2 & 1.1 & 0.08 & 0.00 \\
$\partial M_{2} / \partial \kappa_{3}$ & 3.2 & 0.6 & 0.05 & 0.00 \\
$\partial M_{3} / \partial \kappa_{3}$ & 2.0 & 2.8 & 0.13 & 0.00 \\
\hline flops & 2794 & 3269 & 4755 & $\mathbf{1 4 5 8 2}$ \\
\hline
\end{tabular}

hand, considerably larger (up to $16 \%$ ). If we use the 3-point Gaussian integration, the error is - surprisingly - even much greater (44\%). Such an error in the cross-section tangent stiffnesses may result in divergence of the global Newton's iteration (see Section 5.4). The 5-point Gaussian integration is found to be rather accurate. The use of 10-point Gaussian integration gives the results accurate to six figures. Table 1 additionally shows the total number of flops required for each type of integration. The analytical integration requires the smallest number of flops; the comparably accurate 10-point numerical in- 
Table 2

Relative error (in per cent) of the numerical results based on area integrals with the decomposition into thin layers.

\begin{tabular}{|c|rrrr|rrrr|}
\hline & \multicolumn{9}{|c|}{ 2-point Gaussian integration } & \multicolumn{4}{|c|}{ 3-point Gaussian integration } \\
\hline & $l=3+1$ & $5+2$ & $8+3$ & $15+3$ & $l=3+1$ & $5+2$ & $8+3$ & $15+3$ \\
\hline$N_{1}$ & 3.0 & 0.3 & 1.0 & 0.3 & 2.8 & 0.2 & 1.0 & 0.3 \\
$M_{2}$ & 0.6 & 0.5 & 1.3 & 0.5 & 0.5 & 0.5 & 1.3 & 0.5 \\
$M_{3}$ & 15.0 & 2.6 & 3.9 & 1.6 & 14.8 & 2.6 & 3.9 & 1.6 \\
$\partial N_{1} / \partial \gamma_{1}$ & 34.0 & 1.6 & 4.7 & 1.3 & 13.0 & 0.8 & 4.6 & 1.3 \\
$\partial N_{1} / \partial \kappa_{2}$ & 3.4 & 1.4 & 1.0 & 0.3 & 4.5 & 1.6 & 0.9 & 0.3 \\
$\partial N_{1} / \partial \kappa_{3}$ & 29.1 & 15.6 & 0.5 & 0.4 & 25.3 & 15.0 & 0.4 & 0.4 \\
$\partial M_{2} / \partial \kappa_{2}$ & 4.6 & 0.5 & 1.6 & 0.7 & 4.3 & 0.5 & 1.6 & 0.7 \\
$\partial M_{2} / \partial \kappa_{3}$ & 23.0 & 2.2 & 3.8 & 1.8 & 23.6 & 2.2 & 3.8 & 1.8 \\
$\partial M_{3} / \partial \kappa_{3}$ & 5.5 & 13.0 & 3.3 & 1.9 & 6.5 & 13.0 & 3.3 & 1.9 \\
\hline flops & 1095 & 1619 & 2408 & 3753 & 1476 & 2093 & 3008 & 4584 \\
\hline
\end{tabular}

tegration requires about 5-times more operations; a fairly accurate solution is provided by the 5-point integration but the operations count is again not in its favour.

Table 2 shows the errors of the layer approach. We used larger number of layers in compression than in tension. Results in Table 2 were first obtained by 2point Gaussian integration in the non-uniform direction, and next by 3-point Gaussian integration. The main advantage of this method is a low number of floating point operations. The relative error, however, is considerable when using a low number of layers. With 8 layers in compression and 3 in tension the results compare well to the analytical ones, with the number of flops being almost the same as in the analytical method. To reduce any error below $2 \%$, we again need more floating point operations as in the analytical case. The order of Gaussian integration is not found to be influential, except possibly for the low number of layers. For example, the use of 5-point Gaussian integration gives the same results as the 3-point integration for the range of the number of layers given in Table 2 .

The method given in Section 4.3 is interesting because of its generality and simplicity. Unfortunately, such an approach demands an extremely large number of flops (Table 3). Moreover, the error of the method is diminishing more slowly than in the previous cases. The reason for this is a relatively large number of integration points outside the integration area, which turns out to be even more pronounced in our next example. 
Table 3

Relative error (in per cent) of the numerical results based on 2-dimensional Gaussian area integrals.

\begin{tabular}{|c|rrrr|}
\hline & $n=5 \times 5$ & $n=10 \times 10$ & $n=20 \times 20$ & $n=30 \times 30$ \\
\hline$N_{1}$ & 18.4 & 1.7 & 0.0 & 1.9 \\
$M_{2}$ & 19.1 & 1.5 & 0.4 & 2.1 \\
$M_{3}$ & 58.5 & 0.5 & 1.3 & 3.2 \\
$\partial N_{1} / \partial \gamma_{1}$ & 98.6 & 11.1 & 9.7 & 9.5 \\
$\partial N_{1} / \partial \kappa_{2}$ & 15.2 & 3.2 & 0.2 & 1.8 \\
$\partial N_{1} / \partial \kappa_{3}$ & 57.6 & 0.0 & 10.1 & 3.7 \\
$\partial M_{2} / \partial \kappa_{2}$ & 19.2 & 2.0 & 0.4 & 2.4 \\
$\partial M_{2} / \partial \kappa_{3}$ & 44.7 & 5.5 & 1.9 & 4.4 \\
$\partial M_{3} / \partial \kappa_{3}$ & 42.6 & 0.8 & 4.3 & 6.4 \\
\hline flops & 12727 & 68115 & 406690 & 1207586 \\
\hline
\end{tabular}

\subsection{Rectangular cross-section with rectangular hole}

It is of an utmost importance to consider the hollow cross-sections properly. Here we take a simple rectangle with a rectangular hole (Fig. 4a). The proposed strains are:

$$
\gamma_{1}=-0.0010, \quad \kappa_{2}=-0.0001, \quad \kappa_{3}=0.00005
$$

Please observe the numbering of the vertices of the cross-section, which is in the counter-clockwise direction for external, and in the clockwise direction for the internal boundary, to define properly the orientation of the boundary curve.

The exact results and the comparisons with the numerically integrated path integrals are shown in Table 4 . You may see that a very accurate numerical integration, i.e. the 10-point Gaussian integration, requires almost three times more operations as the analytical method. In contrast to our previous example, the low order numerical integration introduces an enormous relative error. Thus, the low order methods are not sufficient, while the higher order methods are too expensive.

The layers method proves to be more successful regarding the accuracy and the efficiency compared to the boundary numerical integration (Table 5). The number of floating-point operations for very accurate results (15 layers in compression and 3 in tension) is again larger than in the analytical case; a $(8+3)$-layer solution combined with the 2-point Gaussian integration needs 3472 flops, which is somewhat less than in the exact solution (3734). The use of two-dimensional integrals in Table 6 demonstrates both the higher numbers of floating-point operations needed and slow convergence of the method. 


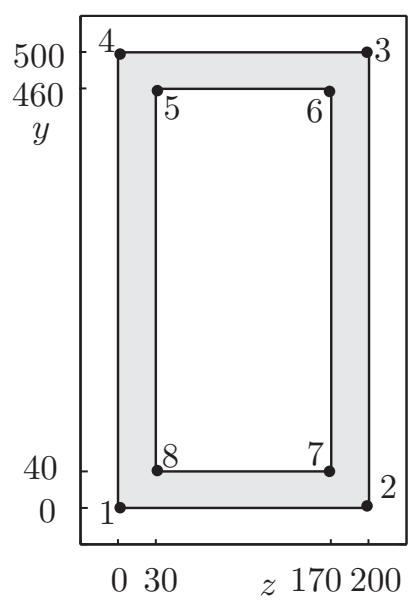

a)

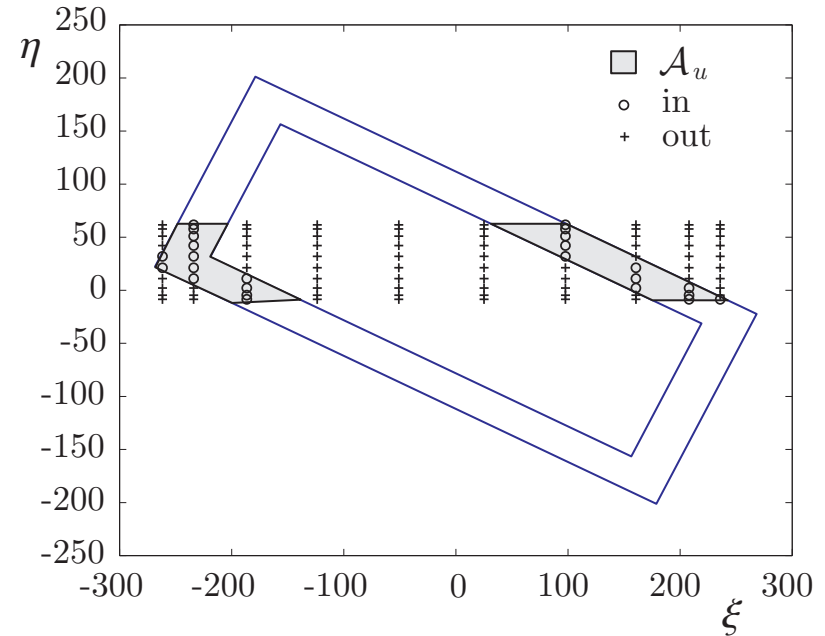

b)

Fig. 4. a) Geometry of the cross-section and b) Gaussian points for the area integral over $\mathcal{A}_{u}$.

Table 4

Analytical results for the cross-section in Fig. 4 (left) and relative error (in per cent) of the numerical results based on Gaussian path integral along boundary (right).

\begin{tabular}{|c|c|}
\hline quantity & value \\
\hline$N_{1}$ & $-2.13 \cdot 10^{4}$ \\
$M_{2}$ & $-2.13 \cdot 10^{5}$ \\
$M_{3}$ & $7.35 \cdot 10^{5}$ \\
$\partial N_{1} / \partial \gamma_{1}$ & $2.24 \cdot 10^{6}$ \\
$\partial N_{1} / \partial \kappa_{2}$ & $-2.67 \cdot 10^{7}$ \\
$\partial N_{1} / \partial \kappa_{3}$ & $1.04 \cdot 10^{8}$ \\
$\partial M_{2} / \partial \kappa_{2}$ & $1.76 \cdot 10^{10}$ \\
$\partial M_{2} / \partial \kappa_{3}$ & $3.78 \cdot 10^{10}$ \\
$\partial M_{3} / \partial \kappa_{3}$ & $6.94 \cdot 10^{10}$ \\
\hline flops & 3734 \\
\hline
\end{tabular}

\begin{tabular}{|c|rrrr|}
\hline & $n=2$ & $n=3$ & $n=5$ & $n=10$ \\
\hline$N_{1}$ & 4.4 & 0.0 & 0.01 & 0.00 \\
$M_{2}$ & 46.7 & 3.1 & 0.00 & 0.00 \\
$M_{3}$ & 30.4 & 0.4 & 0.08 & 0.00 \\
$\partial N_{1} / \partial \gamma_{1}$ & 24.1 & 13.3 & 1.08 & 0.02 \\
$\partial N_{1} / \partial \kappa_{2}$ & 168.7 & 137.2 & 8.03 & 0.15 \\
$\partial N_{1} / \partial \kappa_{3}$ & 113.6 & 66.2 & 3.62 & 0.09 \\
$\partial M_{2} / \partial \kappa_{2}$ & 10.7 & 14.6 & 0.99 & 0.02 \\
$\partial M_{2} / \partial \kappa_{3}$ & 21.7 & 13.6 & 0.86 & 0.02 \\
$\partial M_{3} / \partial \kappa_{3}$ & 35.9 & 14.2 & 0.78 & 0.03 \\
\hline flops & 4005 & 4584 & 6278 & 11143 \\
\hline
\end{tabular}

From Fig. 4b it is obvious that the majority of integration points are situated outside the cross-sectional area. As observed from Fig. 4b, this method can straightforwardly be modified such that the integration is spread only over closed subregions $\mathcal{A}_{u}$ and $\mathcal{A}_{m}$. Such extension was not implemented in the course of this article. 
Table 5

Relative error (in per cent) of the numerical results based on the thin layers area integrals.

\begin{tabular}{|c|rrrr|rrrr|}
\hline & \multicolumn{9}{|c|}{ 2-point Gaussian integration } & \multicolumn{4}{|c|}{ 3-point Gaussian integration } \\
\hline & $l=3+1$ & $5+2$ & $8+3$ & $15+3$ & $l=3+1$ & $5+2$ & $8+3$ & $15+3$ \\
\hline$N_{1}$ & 0.5 & 0.1 & 0.00 & 0.03 & 0.2 & 0.1 & 0.00 & 0.03 \\
$M_{2}$ & 6.2 & 2.9 & 0.15 & 0.35 & 6.6 & 2.9 & 0.15 & 0.35 \\
$M_{3}$ & 2.8 & 1.3 & 0.13 & 0.27 & 2.7 & 1.4 & 0.13 & 0.27 \\
$\partial N_{1} / \partial \gamma_{1}$ & 7.6 & 0.3 & 0.00 & 0.04 & 0.9 & 0.1 & 0.01 & 0.04 \\
$\partial N_{1} / \partial \kappa_{2}$ & 88.2 & 28.4 & 9.60 & 2.97 & 79.8 & 27.0 & 9.45 & 2.96 \\
$\partial N_{1} / \partial \kappa_{3}$ & 39.3 & 13.3 & 4.92 & 1.59 & 39.6 & 13.5 & 4.92 & 1.58 \\
$\partial M_{2} / \partial \kappa_{2}$ & 7.9 & 0.7 & 0.04 & 0.04 & 0.1 & 0.4 & 0.03 & 0.04 \\
$\partial M_{2} / \partial \kappa_{3}$ & 6.5 & 0.3 & 0.15 & 0.02 & 0.9 & 0.0 & 0.13 & 0.02 \\
$\partial M_{3} / \partial \kappa_{3}$ & 5.7 & 0.1 & 0.27 & 0.01 & 2.2 & 0.4 & 0.26 & 0.01 \\
\hline flops & 1593 & 2396 & 3472 & 5383 & 1974 & 2870 & 4072 & 6214 \\
\hline
\end{tabular}

Table 6

Relative error (in per cent) of the numerical results based on 2-dimensional Gaussian area integrals.

\begin{tabular}{|c|rrrr|}
\hline & $n=5 \times 5$ & $n=10 \times 10$ & $n=20 \times 20$ & $n=30 \times 30$ \\
\hline$N_{1}$ & 8.9 & 0.5 & 2.5 & 0.5 \\
$M_{2}$ & 116.5 & 21.5 & 10.7 & 4.8 \\
$M_{3}$ & 34.6 & 9.4 & 1.1 & 3.4 \\
$\partial N_{1} / \partial \gamma_{1}$ & 15.5 & 22.4 & 7.6 & 3.2 \\
$\partial N_{1} / \partial \kappa_{2}$ & 438.2 & 35.4 & 32.2 & 32.1 \\
$\partial N_{1} / \partial \kappa_{3}$ & 148.2 & 4.7 & 11.3 & 16.0 \\
$\partial M_{2} / \partial \kappa_{2}$ & 40.8 & 18.2 & 6.3 & 2.3 \\
$\partial M_{2} / \partial \kappa_{3}$ & 34.8 & 17.6 & 5.6 & 1.8 \\
$\partial M_{3} / \partial \kappa_{3}$ & 39.3 & 19.6 & 6.7 & 1.3 \\
\hline flops & 19886 & 80812 & 448995 & 1302769 \\
\hline
\end{tabular}

\subsection{Trapezoidal cross-section with trapezoidal hole}

This example is both interesting and demanding. We chose a non-rectangular cross-section with the trapezoidal hole (Fig. 5a). Such a cross-section is typical in reinforced or prestressed concrete bridges. We took the following strains:

$$
\gamma_{1}=-0.00050, \quad \kappa_{2}=0.00001, \quad \kappa_{3}=0.00001
$$


Table 7

Analytical results for the cross-section in Fig. 5 (left) and relative error (in per cent) of the numerical results based on Gaussian path integral along boundary (right).

\begin{tabular}{|c|c|}
\hline quantity & value \\
\hline$N_{1}$ & $-7.72 \cdot 10^{4}$ \\
$M_{2}$ & $2.43 \cdot 10^{6}$ \\
$M_{3}$ & $-1.43 \cdot 10^{7}$ \\
$\partial N_{1} / \partial \gamma_{1}$ & $1.11 \cdot 10^{7}$ \\
$\partial N_{1} / \partial \kappa_{2}$ & $-4.07 \cdot 10^{8}$ \\
$\partial N_{1} / \partial \kappa_{3}$ & $-1.69 \cdot 10^{9}$ \\
$\partial M_{2} / \partial \kappa_{2}$ & $2.17 \cdot 10^{11}$ \\
$\partial M_{2} / \partial \kappa_{3}$ & $2.71 \cdot 10^{11}$ \\
$\partial M_{3} / \partial \kappa_{3}$ & $-1.20 \cdot 10^{11}$ \\
\hline flops & 4318 \\
\hline
\end{tabular}

\begin{tabular}{|c|rrrr|}
\hline & $n=2$ & $n=3$ & $n=5$ & $n=10$ \\
\hline$N_{1}$ & 0.6 & 0.9 & 0.02 & 0.00 \\
$M_{2}$ & 7.2 & 0.7 & 0.04 & 0.00 \\
$M_{3}$ & 10.2 & 0.4 & 0.02 & 0.00 \\
$\partial N_{1} / \partial \gamma_{1}$ & 66.2 & 6.2 & 0.08 & 0.00 \\
$\partial N_{1} / \partial \kappa_{2}$ & 39.5 & 15.5 & 0.50 & 0.00 \\
$\partial N_{1} / \partial \kappa_{3}$ & 59.9 & 20.0 & 0.57 & 0.00 \\
$\partial M_{2} / \partial \kappa_{2}$ & 27.4 & 4.3 & 0.01 & 0.00 \\
$\partial M_{2} / \partial \kappa_{3}$ & 14.3 & 3.1 & 0.17 & 0.00 \\
$\partial M_{3} / \partial \kappa_{3}$ & 156.9 & 1.6 & 0.92 & 0.00 \\
\hline flops & 4605 & 5230 & 7016 & 17593 \\
\hline
\end{tabular}

The analytical results are shown in Table 7 . The comparison with numerical results is shown in Tables 7, 8 and 9. The conclusions drawn from the previous two examples can again be confirmed. The sufficiently high-order numerical boundary integration gives accurate, but rather expensive results. The layer-based integration is relatively inexpensive, but still demands more floating-point operations than the analytical one to obtain sufficiently accurate results. The area integrations as presented in Section 4.3 again turns out to be computationaly very demanding.

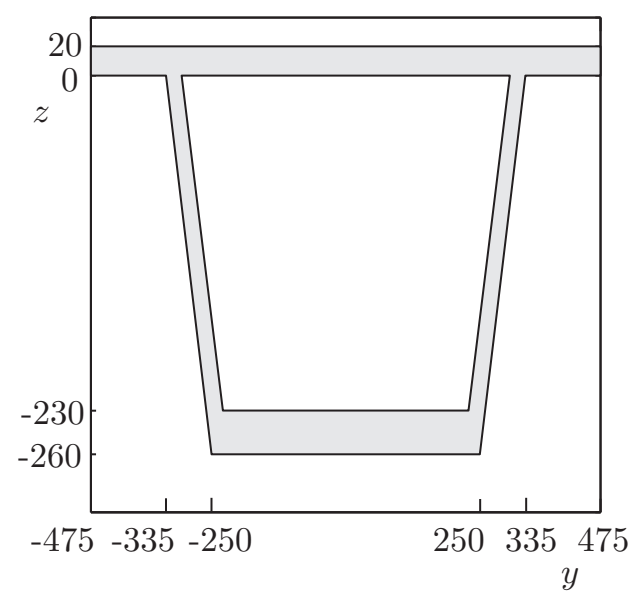

a)

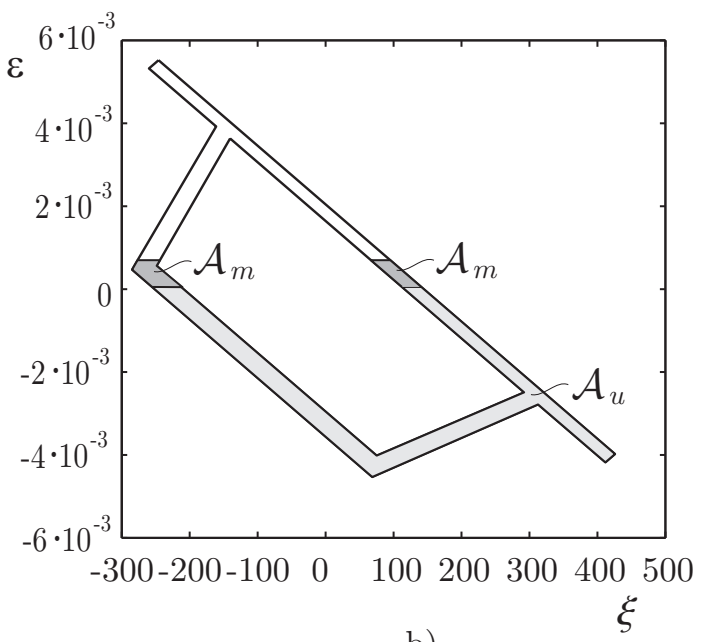

b)

Fig. 5. a) Geometry of the trapezoidal section and b) its image in the coordinate system $(\xi, \varepsilon)$ with subregions $\mathcal{A}_{u}$ and $\mathcal{A}_{m}$. 
Table 8

Relative error (in per cent) of the numerical results based on area integrals with decomposition into thin layers.

\begin{tabular}{|c|c|c|c|c|c|c|c|c|}
\hline & \multicolumn{4}{|c|}{ 2-point Gaussian integration } & \multicolumn{4}{|c|}{ 3-point Gaussian integration } \\
\hline & $l=3+1$ & $5+2$ & $8+3$ & $15+3$ & $l=3+1$ & $5+2$ & $8+3$ & $15+3$ \\
\hline$N_{1}$ & 3.2 & 3.15 & 2.8 & 0.18 & 3.2 & 3.2 & 2.82 & 0.18 \\
\hline$M_{2}$ & 43.1 & 27.34 & 11.1 & 1.74 & 43.1 & 27.3 & 11.10 & 1.74 \\
\hline$M_{3}$ & 4.7 & 3.73 & 5.6 & 0.23 & 4.7 & 3.7 & 5.58 & 0.23 \\
\hline$\partial N_{1} / \partial \gamma_{1}$ & 5.6 & 3.55 & 2.1 & 0.11 & 5.7 & 3.5 & 2.06 & 0.11 \\
\hline$\partial N_{1} / \partial \kappa_{2}$ & 68.3 & 32.25 & 12.3 & 2.70 & 66.8 & 32.1 & 12.23 & 2.70 \\
\hline$\partial N_{1} / \partial \kappa_{3}$ & 1.6 & 2.96 & 6.1 & 0.01 & 1.1 & 2.9 & 6.08 & 0.01 \\
\hline$\partial M_{2} / \partial \kappa_{2}$ & 6.6 & 4.14 & 0.5 & 0.27 & 6.5 & 4.1 & 0.50 & 0.27 \\
\hline$\partial M_{2} / \partial \kappa_{3}$ & 4.0 & 5.87 & 3.6 & 0.48 & 4.0 & 5.9 & 3.63 & 0.48 \\
\hline$\partial M_{3} / \partial \kappa_{3}$ & 76.1 & 29.19 & 25.4 & 1.66 & 77.0 & 29.3 & 25.37 & 1.66 \\
\hline flops & 1612 & 2407 & 3469 & 5508 & 1993 & 2881 & 4069 & 6339 \\
\hline
\end{tabular}

Table 9

Relative error (in per cent) of the numerical results based on 2-dimensional Gaussian area integrals.

\begin{tabular}{|c|rrrr|}
\hline & $n=10 \times 10$ & $n=30 \times 30$ & $n=50 \times 50$ & $n=100 \times 100$ \\
\hline$N_{1}$ & 8.8 & 5.4 & 0.2 & 0.2 \\
$M_{2}$ & 53.0 & 25.0 & 3.0 & 0.4 \\
$M_{3}$ & 13.4 & 3.5 & 1.2 & 0.6 \\
$\partial N_{1} / \partial \gamma_{1}$ & 22.5 & 1.2 & 2.6 & 2.0 \\
$\partial N_{1} / \partial \kappa_{2}$ & 133.8 & 21.6 & 7.1 & 2.8 \\
$\partial N_{1} / \partial \kappa_{3}$ & 3.0 & 11.7 & 1.5 & 0.1 \\
$\partial M_{2} / \partial \kappa_{2}$ & 8.9 & 1.9 & 2.4 & 1.8 \\
$\partial M_{2} / \partial \kappa_{3}$ & 0.8 & 3.1 & 2.1 & 1.2 \\
$\partial M_{3} / \partial \kappa_{3}$ & 2.6 & 13.6 & 11.6 & 4.4 \\
\hline flops & 78280 & 1301028 & 5126778 & 34786829 \\
\hline
\end{tabular}

\subsection{Reinforced concrete cantilever under free-end force}

In our last example we show the effect of the insufficiently accurate integration over the cross-section on the convergence of the non-linear finite-element analysis of a reinforced concrete beam. The beam is clamped at one end and subjected to the force $F_{Z}=6.5 \mathrm{kN}$ at the other (Fig. 6). The force is applied in a single step.

The geometric data of the cantilever are:

$$
L=150 \mathrm{~cm}, \quad h=20 \mathrm{~cm}, \quad t=10 \mathrm{~cm} .
$$




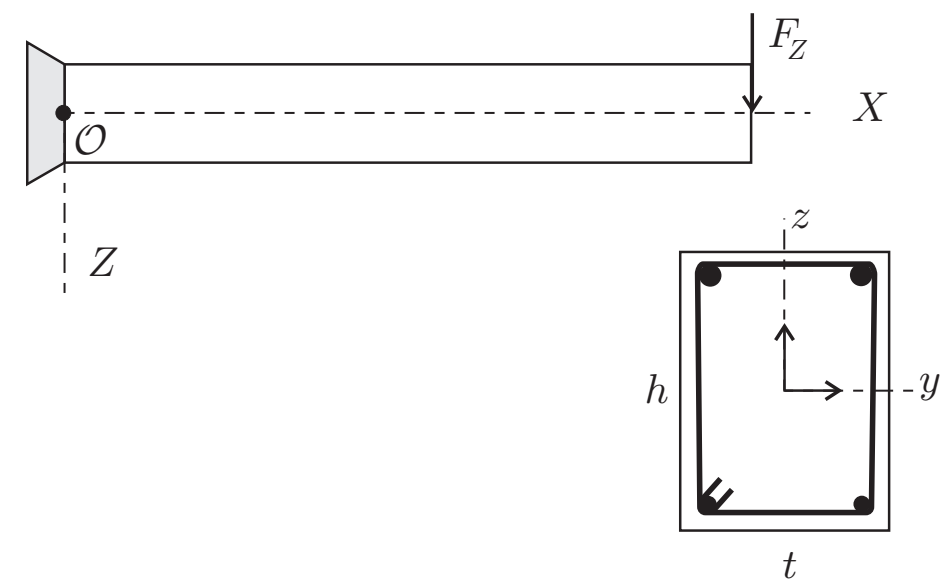

Fig. 6. Reinforced concrete cantilever.

The same parameters of concrete are taken as in the previous numerical examples. The reinforcement consists of four steel bars with one bar in each corner of the rectangular cross-section. The diameter of the bars is $1.6 \mathrm{~cm}$ at the top of the cross-section and $0.8 \mathrm{~cm}$ at the bottom. The centroids of the bars are $3 \mathrm{~cm}$ away from the edge. A linear elastic material is assumed for steel reinforcement with elastic modulus $E_{\mathrm{s}}=20000 \mathrm{kN} / \mathrm{cm}^{2}$.

We used a single 5-node strain-based beam finite element [13] for the determination of the equilibrated deformed state of the cantilever. We employed both the analytical and the numerical 2-dimensional integration of various orders (Section 4.3). The results for the free-end displacement in the direction of the applied force and the corresponding number of iterations needed in the global Newton's iteration are shown in Table 10. There we show how the accuracy of the cross-sectional integration effects the number of iterations. As a rule, less iterations are required when an inaccurate low order numerical integration is employed, but the results tend to be less accurate, and in some cases $(N=2$ and $N=4$ ), the iteration does not converge at all, inspite of the fact that the residual vector and the tangent stiffness matrix in the formulation [13] are fully consistent. Thus, the errors in the residual vector and the tangent stiffness matrix have either a beneficial effect (i.e. a faster convergence) or, in some hardly identifiable cases, cause the divergence. A similar conclusion was found in [12] for the elastic-plastic analysis of beams.

\section{Conclusions}

We presented a procedure for the analytical integration of the stress field and material moduli over the concrete cross-section. The essential points of the 
Table 10

Number of global Newton's iterations.

\begin{tabular}{|c|c|c|c|c|c|c|c|c|}
\hline & \multirow{2}{*}{ analytical } & \multicolumn{7}{|c|}{ numerical 2-dimensional integration, $n \times n$} \\
\hline & & $2 \times 2$ & $3 \times 3$ & $4 \times 4$ & $5 \times 5$ & $10 \times 10$ & $20 \times 20$ & $100 \times 100$ \\
\hline$n_{i t}$ & 8 & $\infty$ & 4 & $\infty$ & 5 & 5 & 6 & 8 \\
\hline$u_{Z}$ & 0.06549 & - & 0.06717 & - & 0.06521 & 0.06398 & 0.06548 & 0.06549 \\
\hline
\end{tabular}

present approach are:

1. The analytical approach is preferable, because it gives exact values. In contrast, the numerical integration is only approximative, and requires a sufficient number of integration points, which is not known a priori, to achieve a sufficient accuracy. This usually requires the computational time which is greater than the one, needed for an analytical integration.

2. Analytical integrals can be prepared in advance (formal definite integrals of the prescribed constitutive law), which further increases the efficiency of the integration algorithm.

3. Analytical integrals were prepared for the constitutive law proposed by Desayi and Krishnan [4] and Bergan and Holand [1]. Any constitutive law prescribed in an analytical form can be employed, provided that the integrals in Eq. (6) can be integrated analytically. One of such laws is given in Eurocode 5 [5].

4. The method is here applied only for the case when the strain distribution over the cross-section is linear. In principle, however, the method is applicable for any smooth strain distribution uniform in one direction.

5. The proposed method is directly applicable in various existing materially non-linear beam formulations, provided that the strain-reversals in the crosssection do not occur, much alike as in the serviceability and ultimate limit state studies of structures.

\section{References}

[1] P. G. Bergan, I. Holand, "Nonlinear finite element analysis of concrete structures", Comput. Methods Appl. Mech. Engrg. 17/18, 443-467, 1979.

[2] J. L. Bonet, P. F. Miguel, M. L. Romero, M. A. Fernández, "A modified algorithm for reinforced concrete cross section integration", In Proceedings of the Sixth International Conference on Computational Structures Technology, B.H.V. Topping (editor), Civil-Comp Press, article 120, 2002. 
[3] T. Brøndum-Nielsen, "Serviceability analysis of concrete sections under biaxial bending", ASCE J. Struct. Engrg. 123(1), 117-119, 1997.

[4] P. Desayi, S. Krishnan, "Equation for the stress-strain curve of concrete", J. Am. Concrete Inst. 61, 345-350, 1964.

[5] Eurocode 2, Design of concrete structures. Part 1: General rules and rules for buildings, prEN 1992-1: 2001, 1st draft, 1999.

[6] Matlab, The language of technical computing, The Mathworks, Inc., 1999.

[7] S. E. El-Metwally, A. M. El-Shahhat, W. F. Chen, "3-D nonlinear analysis of R/C slender columns", Comput. Struct. 37, 863-872, 1990.

[8] A. Fafitis, "Interaction surfaces of reinforced-concrete sections in biaxial bending", ASCE J. Struct. Engrg. 127, 840-846, 2001.

[9] B. A. Izzuddin, A. A. F. M. Siyam, D. Lloyd Smith, An efficient beam-column formulation for $3 D$ reinforced concrete frames, Comput. Struct., 80, 659-676, 2002.

[10] H. A. S. Rasheed, K. S. Dinno, "An efficient nonlinear analysis of RC sections", Comput. Struct. 53, 613-623, 1994.

[11] J. A. Rodriguez, J. D. Aristizabal-Ochoa, "Biaxial interaction diagrams for short RC columns of any cross-section", ASCE J. Struct. Engrg. 125(6), 672683, 1999.

[12] M. Saje, I. Planinc, G. Turk, B. Vratanar, "A kinematically exact finite element formulation of planar elastic-plastic frames", Comput. Methods Appl. Mech. Engrg. 144, 125-151, 1997.

[13] D. Zupan, M. Saje, "Finite-element formulation of geometrically exact threedimensional beam theories based on interpolation of strain measures", Comput. Methods Appl. Mech. Engrg. 192, 5209-5248, 2003. 